\title{
An Automatic Approach of Road Regions Extraction from Satellite Images based on Connected Component Algorithm
}

\author{
Mudit Shrivastava $^{1}$, Dr. D. M. Bhalerao ${ }^{2}$ \\ ${ }^{1}$ PG Student, dept. of E\&TC, Sinhgad College of Engineering, Vadgaon (BK), Pune, Savitribai PhulePune University, Pune, India \\ ${ }^{2}$ Professor, dept. of E\&TC, Sinhgad College of Engineering, Vadgaon (BK), Pune, Savitribai Phule Pune University, Pune, India
}

\begin{abstract}
Satellite images provide valuable information for a wide range of applications. They use various spectral bands to recognize a great number of features. High-resolution satellite (HRS) images are able to represent man-made features such as roads and buildings. Roads are the most fundamental structures. We have presented a methodology using image processing and connected component method for automatic extraction of road network from any high resolution satellite image. It is sometimes challenging in the areas having complex scenes. There is an algorithm to extract the manmade features from the image and filtering operations are used to correctly extract out the respective feature i.e. the road network from the image. The proposed system follows various steps consisting of Enhancement, Filtering operations, Segmentation etc. The final extracted road network can be used for the map creation which shortens the human efforts in creating large scale maps and also it is very efficient in time and cost. The method is reliable to implement in real time also and can be applied for the real time development in the field of remote sensing and Geographic Information System including rural and urban development. The algorithm promises to provide good results and helpful in keeping Geographical Information System (GIS) updated with new road databases by reducing the time consumption in producing large scale maps.
\end{abstract}

Keywords: Connected component, Enhancement, Feature extraction, Filtering operations, Road network detection

\section{Introduction}

The aim of our road network extraction process is just to provide a binary form of the concerned satellite image which consists of two regions i.e. true region representing the road region and the false region representing non-road regions. The road detection methods which are requiring human interaction are classified as semi-automatic, and those that are not requiring human interaction are known as automatic. Extraction and detection of Road networks from satellite images is an important research subject and it is mostly used for various Remote sensing applications. It basically generates geospatial information for large areas with very minor inaccuracy. Moreover cost and time of data production using these methods are far lower than almost all other production methods. Roads recognition is unavoidable as they form an important Geographic Information System (GIS) layer in remarkable civilian and military applications involving navigation or Location aware systems and emergency planning systems for evacuation and Fire response [1].

Primarily improvement of pictorial information for human interpretation is one of the major application areas in image processing. A number of satellite original images contain noise. This can be eliminated by several enhancement techniques. Filtering is an enhancement technique, used to remove or filter unnecessary information (noise) from the image. It is also applied for image smoothening and sharpening. Few neighbourhood operations work with the values of the image pixels in the neighbourhood and the respective values of a sub image having the same dimensions. Picture enhancement and its gray level adjustment are the primary steps towards the fulfilment of the whole process. After converting the image into gray scale image its effects can be analysed using histogram analysis. Using histogram, we can analyse the black and white proportions of the image. Once image is converted into gray scale form, its level is adjusted in such a manner that black and white proportions of the image could be separated out up to a certain level so that thresholding in the next step might become easier as our primary task is to provide approximate binary image before reaching to thresholding. GIS needs the automatic extraction process of roads for their data updating because it is very costly and time consuming process to update data to their database manually also there may be a possibility of error in manual updating of road network.

There are variety of services facilitated by GIS applications containing road databases include satellite navigation, creation and updating of accurate geographical databases, route planning, health-care accessibility planning, military operations, infrastructure management, land cover classification and also the extent of damages after natural disasters such as floods, earthquakes [2]. The aim of road network extraction is just to provide a binary form which consists of two regions i.e. true region representing the road region and the false region representing non-road regions but the problem arises when there are complex structures in the image containing several different objects, such as roads, trees, vehicles, houses, etc.., with differences in their shapes and textures.

An image contains number of connected components (CC), and each $\mathrm{CC}$ is a set of pixels where each pair of pixels is connected to one another. There is also a method called Trivial opening which is used here to filter these sets of CC of the image based on particular conditions. If this specific condition is true then connected component is retained 


\section{International Journal of Science and Research (IJSR) \\ ISSN (Online): 2319-7064}

Index Copernicus Value (2013): 6.14 | Impact Factor (2015): 6.391

otherwise, it is removed. We may use trivial opening only when the concerned image is having the connected components $[3,4]$.

Proposed method can be applied on noisy images too, and good results can be obtained. In this paper we have presented an algorithm based on connected component for road network extraction, Secondsection of this paperprovides proposed methodology and algorithm, third section relates with connected component algorithm. In the final sections we can conclude the paper after providing the experimental setup and the obtained results.

\section{Proposed Methodology}

The methodology of the road network extraction includes several steps. In this a satellite image is loaded first then various enhancement methods are performed to improve the quality of the image before proceeding to the next steps. After that, image is converted into gray scale image. Subsequently other operations are performed as given in the figure 1 as per the complexity and noise level of input image loaded

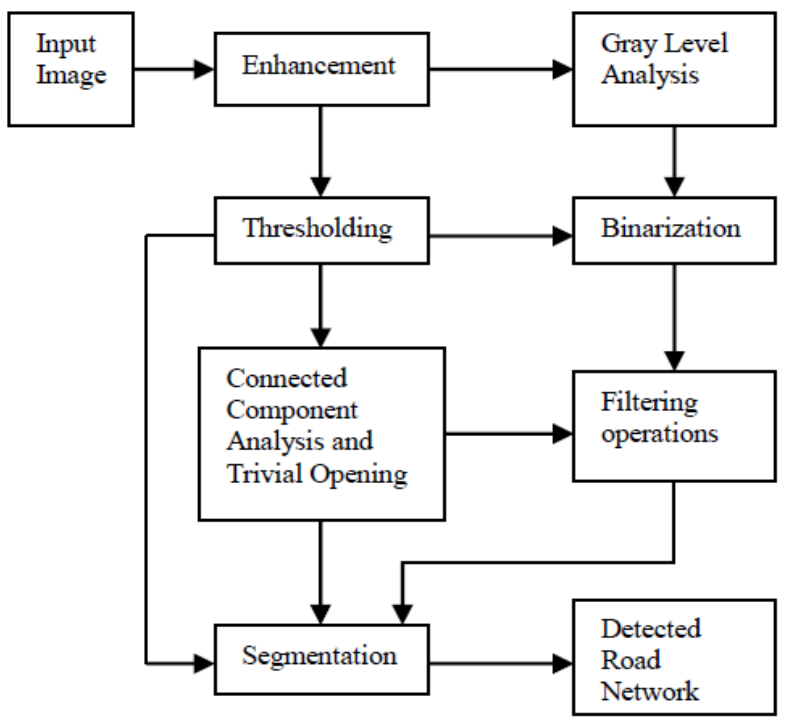

Figure 1: Road Network Extraction Algorithm

The figure 1 illustrates the road network extraction algorithm which includes various steps which are performed over the input test satellite image. The details of each step are presented below.

I. The input image is loaded. It is called the test image and its pixel intensity is analysed by obtaining its histogram, shown in figure 2 and figure 3.

Now Image is enhanced by applying the enhancement step and its aim is to improve the visual effects in the image. Thus it helps geographic image interpretation and improves the contrast and brightness between the target (road regions) and non-target (non-road regions) for high level image processing. Enhancement operation causes in the internal regions of the manmade structures being more homogeneous than the border regions of the image [1].

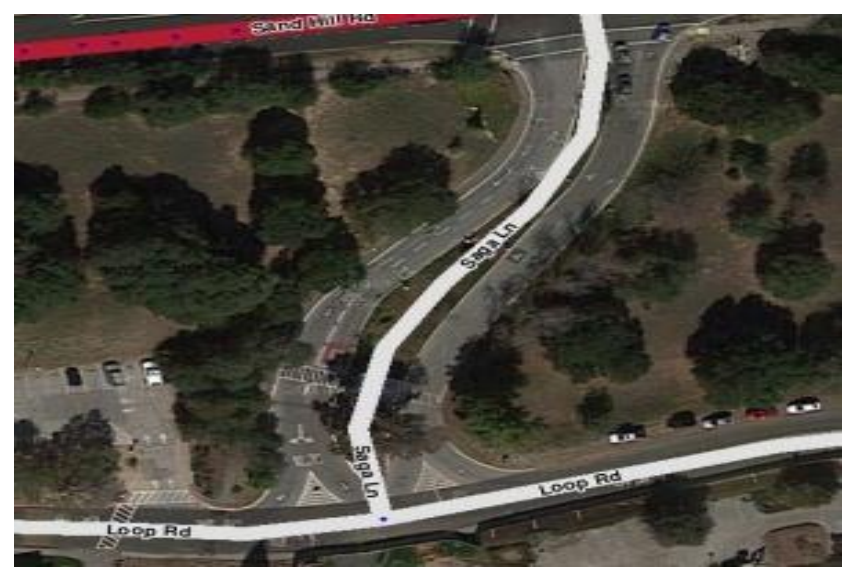

Figure 2: Input image

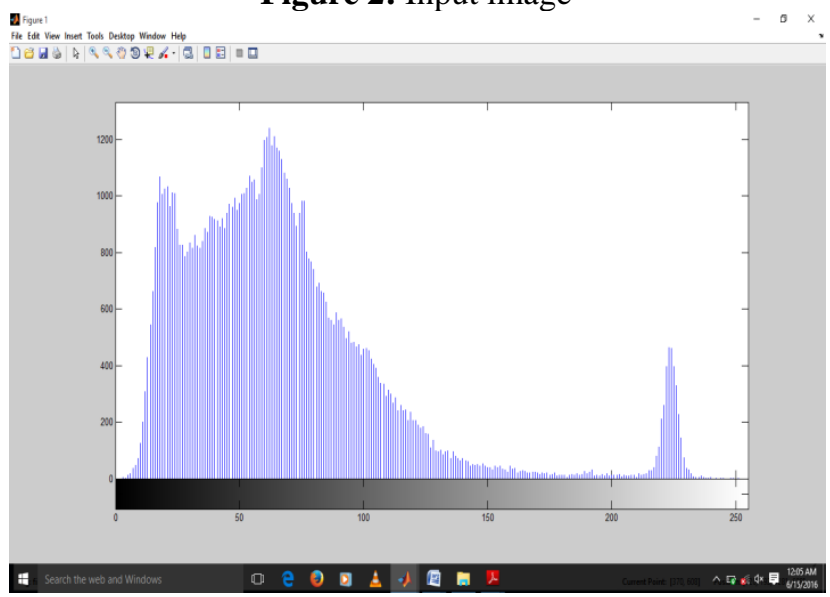

Figure 3: Image Histogram of Input image

II. Now after the application of enhancement method the manmade objects appear more prominent in the image than the natural objects. Hence it facilitates now to apply gray scale conversion on the enhanced image because it contains fewer natural objects as per our need and image is converted to gray scale form. [1]

III. The next step is binarization in which gray scale image is converted to binary image or black and white image and it can also yield to thresholding. Adaptive global thresholding is applied here to remove the non-road pixels and segment approximated road region from the satellite image[5]. Meanwhile if image contains noise then filtering operation can be performed to remove the noise from the image.

Thresholding is applied on the binary image. A suitable threshold value is selected to segment the approximated road regions from the image. Otsu's algorithm is applied to find the threshold value $V_{T}$ which is useful to segment the road region pixels $r(x, y)$ from the original image pixels $f(x, y)$.

$$
r(x, y)= \begin{cases}o, & \text { if } f(x, y)<V_{T} \\ 1, & \text { if } f(x, y) \geq V_{T}\end{cases}
$$

The histogram shown in the figure 3 of the satellite image is analyzed and categorised into four principle sections to obtain the desired threshold value for the segmentation, The histogram is devided into sections based on the average value $(\mathrm{M})$ of all the pixel intensities and tonal gray values in the image. Section A shows the pixels having the tonal values with toal gray varience from 0 to 60 on $\mathrm{X}$ axis. This section recognizes dark objects such as vehicles, lakes ,shadows etc in the image. Section B relates with the pixels

\section{Volume 5 Issue 6, June 2016} www.ijsr.net 


\section{International Journal of Science and Research (IJSR) \\ ISSN (Online): 2319-7064}

Index Copernicus Value (2013): 6.14 | Impact Factor (2015): 6.391

having tonal values from varience of 60 to 120 . This section provides the information of dark gray shade objects like grasslands, trees, etc. Section $\mathrm{C}$ finds the pixel intensities with tonal variance 120 to 180 , it belongs to bright gray objects for instance roads, lanes, etc. Section D relates with the group of pixels having the tonal gray values 180 to 250 on $\mathrm{X}$ axis and this section finds the bright objects such as bright vehicles, concrete cement road, etc. Adaptive theresholding technique is used in this case because single value thresholding might not work here properly as threshold value of pixel is based on its position within an image. From this technique we can extract approximate road regions [5]. Therefore, this technique is called as adaptive global thresholding. From this technique, approximated road regions are identified. The pixels that lie in that region are assigned to value 1 and all the remaining pixels are made to 0 . Now, gray image is converted into binary image in which road regions appeared in white and all other pixels appeared in black. Thus this particular threshold value for road regions is accepted and applied for extracting the required road regions out which might or might not be complete due to the complexity nature of the image. Thresholded regions further might lead to the application of Connected Component technique.

\section{Connected Component Algorithm}

Connected component of an image may be defined for any pixel in that image, as a set of pixels that is connected to that pixel [6].The set of pixels, not separated by a definite boundary can be featured as connected pixels. Connected component is actually each maximal region of connected pixels . An image is divided into segments by connected components. Connected component approach is applied for feature extraction, edge detection, line detection, road map extraction, etc. We can extract connected components in an image using dilation and intersection operators..

Suppose $\mathrm{Z}$ be an image and connected component in an image is denoted as A; and a known point of A is denoted as $\mathrm{p}$ which is referred as $\mathrm{X} 0$. Then, the expression given below gives all elements of $\mathrm{A}$.

$$
X_{k}=\left(X_{k-1} \oplus B\right) \cap Z
$$

Here $\mathrm{k}$ belongs to positive integers, symbol $\bigoplus$ represents morphological dilation, $\mathrm{C}$ denotes intersection and $\mathrm{B}$ is a suitable structuring element. If $X_{k}=X_{k-1}$, then the algorithm has converged and let $\mathrm{A}=X_{k}$.

The connected components that are based on some criterian are extracted by trivial opening [7]. Suppose $Z$ be an image $\{\mathrm{Z}(\mathrm{n}) \quad \mid \mathrm{n}=1,2,3 \ldots \ldots, \mathrm{N}\} \quad$ is a sequence of connected components in the image $\mathrm{Z}$, and $\mathrm{z}(\mathrm{i})$ is a point in $\mathrm{Z}(\mathrm{i})$. The trivial opening can be expressed with a condition $S$, as follows in equation 2 .

$$
s_{o}(z)=\left\{\begin{array}{c}
Z(i), \text { if } Z(i) \text { satisfies the condition } S \\
\emptyset, \text { otherwise }
\end{array}\right.
$$

Here, $s_{o}(z)$ is the trivial opening with condition $S$ associated. Trivial opening is the method, which is useful to extract the required connected components from the image based on condition $S$ and remove the connected components which do not satisfy the condition $S$. Road regions can be easily identified and extracted using trivial opening. We can extract long feature roads by selecting the condition as the long axes of minimum ellipse for trivial opening mechanism. Using equation given below, we can obtain trivial opening for road detection [7].

$$
R_{O}=\left\{P \mid \begin{array}{c}
\text { Long axis of minimum ellipse } \\
\text { enclosing } P(i)>=S
\end{array}\right\}
$$

Where $P$ is an image and $P(i)$ is a connected component of the image. We may proceed by extracting all the connected components from the image by using equation 1. Out of these connected components, further road components are segmented using the equation 3 . Thus, the resultant image $R_{O}$ after the analysis has the required connected road component which is greater than $S$.

\section{Experimental Results and Discussion}

The proposed method is applied on test image which is a satellite image, collected from Satellite Imaging Corporation and Apollo Mapping [8, 9]. The experiment is performed on the same image and result is obtained after the segmentation using the proposed method and the road network is detected. Corresponding test image and results obtained after implementing adaptive thresholding, connected component analysis and trivial opening are shown in the figure 4. Final road map can be extracted using the filtering operation on the resulting image.

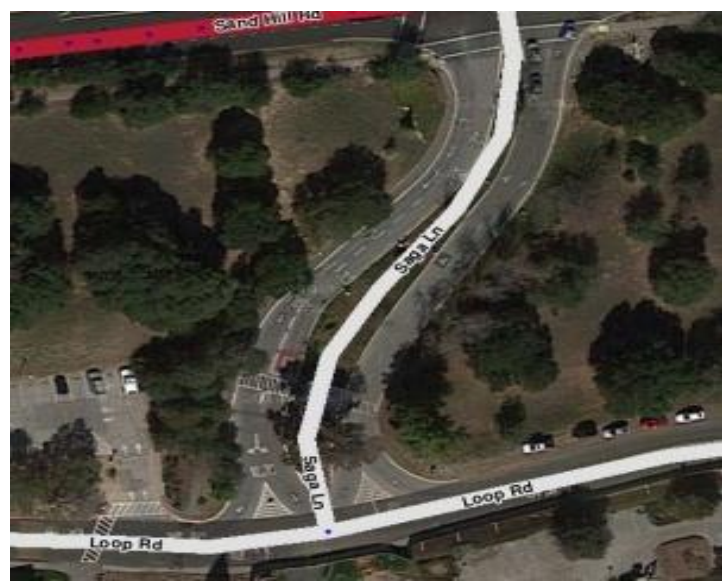

(a)

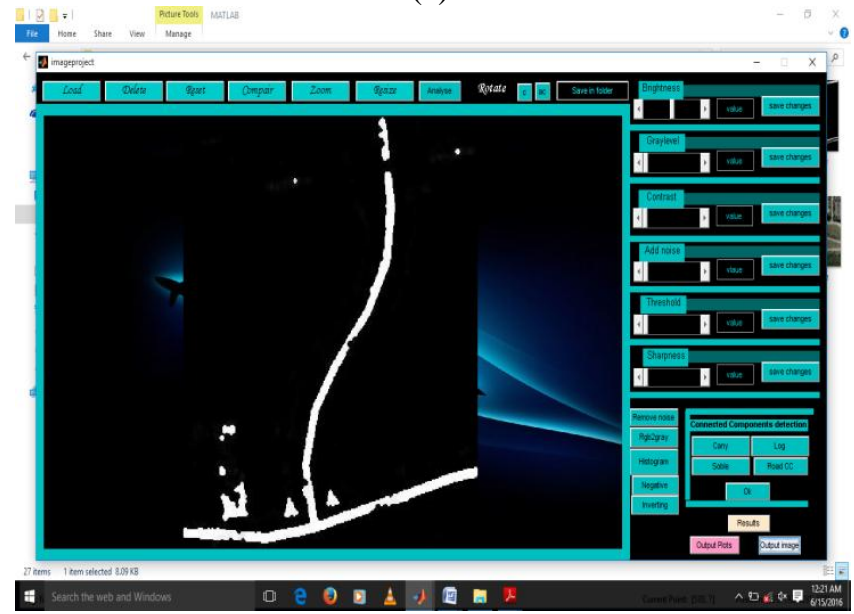

(b)

Figure 4: (a) Input test image (b) Final extracted Road map

Performance of the experiment is analyzed using basically three parameters which are very useful in road network extraction which are known as Completeness, Correctness 


\section{International Journal of Science and Research (IJSR) \\ ISSN (Online): 2319-7064}

Index Copernicus Value (2013): 6.14 | Impact Factor (2015): 6.391

and Quality. These parameters are measured using a ground truth road map which is manually drawn and the extracted road nework is compared with it. We form a buffer around this reference map with constant width.

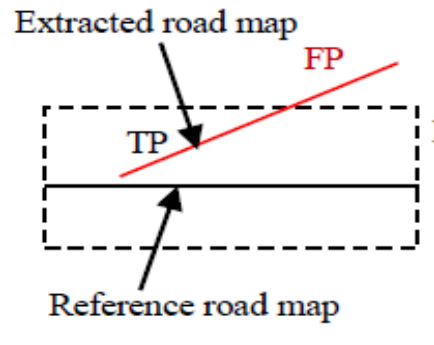

(a)



(b)

Figure 5: (a) Matched Extraction (b) Matched Reference

According to figure 5, the matched road data in extracted map is called true positive (TP) and unmatched road data is False positive (FP). The reference data within buffer is called matched reference. The length of matched portion of reference with extracted road data is TP while unmatched reference is called False Negative (FN) data.

$$
\begin{aligned}
& \text { Compl } \%= \frac{\text { length of the matched reference }}{\text { length of reference }} \\
& \cong \frac{T P}{T P+F N} \times 100 \\
& \text { Correct } \%= \\
& \cong \frac{\text { length of the matched extraction }}{\text { length of extraction }} \\
& \text { Qual } \%= \\
& \text { length of matched extraction } \\
& \text { length of unmatched reference } \\
& \cong \frac{T P}{T P+F P+F N} \times 100
\end{aligned}
$$

Using above equations average measures of the Completeness, Correctness and Quality are determined which are $89 \%, 94 \%$ and $87 \%$ respectively. For evaluation, true positive, false positive and false negtive values can be calculated and counted using manually drawn ground truth map which is obtained using the software GIMP (GNU Image Manipulation Program) [10].

Buffer width is set as 3. Value of TP, FP and FN is counted on the ground truth map as follows.

TP- It is the element which is present on ground truth and the extracted road network both.

FP- It is present on the extracted road nework while not on the ground truth.

FN- It is the element present on ground truth while not on the extracted road network.

Table 1: Comparison of average measuresed values of proposed method with other methods

\begin{tabular}{|c|c|c|c|}
\hline Methods & Compl \% & Correct \% & Qual \% \\
\hline Xiaoying [11] & 78 & 80 & 66 \\
\hline Wenzhong [12] & 79 & 77 & 63 \\
\hline Proposed work & 93 & 88 & 83 \\
\hline
\end{tabular}

Table I shows average measured values of completeness, correctness and quality of the proposed method are compared with other methods and it can be analyzed that proposed method perfomes very well over these methods and good results are obtained using proposed method

\section{Conclusions}

Having up-to-date road maps is crucial for providing many important services. For example, a city requires accurate road maps for routing emergency vehicles, while a GPSbased navigation system needs the same information in order to provide the best directions to its users. Since new roads are constructed frequently keeping road maps up-to-date is an important problem. At present, roadmaps are constructed and updated by hand based on high-resolution aerial imagery. Since very large areas need to be considered, the updating process is costly and time consuming. For this reason automatic detection of roads in high resolution Satellite and aerial imagery has attracted a lot of attention in the remote sensing community. Using proposed algorithm we get very good results of road extraction in terms of Completeness, Correctness and Quality.

\section{References}

[1] D. Chaudhuri, N. K. Kushwaha, and A. Samal, "Semiautomated road detection from high-resolution satellite images by directional morphological enhancement and segmentation techniques," in Proc. IEEE J. Sel. Topics Appl. Earth Observ. Remote Sens., vol. 5, no. 5, pp. 1538-1544, Oct. 2012

[2] N. Lomenie, J. Barbeau, and R. Trias-Sanz, "Integrating texture and geometric information for an automatic bridge detection systems," in Proc. IEEE Int. Geosci. Remote Sens. Symp. (IGARSS), Toulouse, France, Jul. 2003, pp. 3952-3954.

[3] MD Mura, JA Benediktsson, B Waske, L Bruzzone, Morphological attribute profiles for the analysis of very high resolution images. IEEE Trans. Geosci. Remote Sens. 48(10), 3747-3762 (2010)

[4] M Boldt, K Schulza, A Thiele, S Hinz, Using morphological differential attribute profiles for change categorization in high resolution SAR images. Inter. Arch. Photogramm. Remote Sens. Spatial Inf. Sci. 1(1), 29-34 (2013)

[5] PP Singh, RD Garg, Automatic road extraction from high resolution satellite image using adaptive global thresholding and morphological operations. J. Indian Soc. Remote Sens. 41(3), 631-640 (2013)

[6] MD Mura, JA Benediktsson, B Waske, L Bruzzone, Morphological attribute profiles for the analysis of

\section{Volume 5 Issue 6, June 2016 www.ijsr.net}




\section{International Journal of Science and Research (IJSR) \\ ISSN (Online): 2319-7064}

Index Copernicus Value (2013): 6.14 | Impact Factor (2015): 6.391

very high resolution images. IEEE Trans. Geosci. Remote Sens. 48(10), 3747-3762 (2010)

[7] J Serra, L Vincent, An overview of morphological filtering. Circuits, Syst. Signal Proc. 11(1), 47-108 (1992)

[8] Satellite images from Apollo Mapping. Available: https://www.apollomapping.com

[9] Satellite images from Satellite Imaging Corporation (SIC). Available: http://www.satimagingcorp.com

[10] RW Solomon, Free and open source software for the manipulation of digital images. Am. J. Roentgenol. 192(6), 330-334 (2009)

[11] X Jin, CH Davis, An integrated system for automatic road mapping from high-resolution multi-spectral satellite imagery by information fusion. Inf.Fusion 6(4), 257-273 (2005)

[12] W Shi, Z Miao, Q Wang, H Zhang, Spectral-spatial classification and shape features for urban road centerline extraction. IEEE Trans. Geosci. Remote Sens. Lett. 11(4), 788-792 (2014)

Volume 5 Issue 6, June 2016 www.ijsr.net 